\title{
Wave computing with passive memristive networks
}

\author{
Iosif-Angelos Fyrigos*, Vasileios Ntinas ${ }^{* \dagger}$, Georgios Ch. Sirakoulis*, Andrew Adamatzky ${ }^{\ddagger}$, \\ Victor Erokhin ${ }^{\S}$ and Antonio Rubio \\ ${ }^{*}$ Department of Electrical and Computer Engineering, Democritus University of Thrace, Xanthi, Greece \\ ${ }^{\dagger}$ Department of Electronic Engineering, Universitat Polytécnica de Catalunya, Barcelona, Spain \\ ${ }^{\ddagger}$ Unconventional Computing Centre, University of the West of England, Bristol, United Kingdom \\ $\S$ Institute of Materials for Electronics and Magnetism, Parma, Italy
}

\begin{abstract}
Since CMOS technology approaches its physical limits, the spotlight of computing technologies and architectures shifts to unconventional computing approaches. In this area, novel computing systems, inspired by natural and mostly nonelectronic approaches, provide also new ways of performing a wide range of computations, from simple logic gates to solving computationally hard problems. Reaction-diffusion processes constitute an information processing method, occurs in nature and are capable of massive parallel and low-power computing, such as chemical computing through Belousov-Zhabotinsky reaction. In this paper, inspired by these chemical processes and based on the wave-propagation information processing taking place in the reaction-diffusion media, the novel characteristics of the nanoelectronic element memristor are utilized to design innovative circuits of electronic excitable medium to perform both classical (Boolean) calculations and to model neuromorphic computations in the same Memristor-RLC (M-RLC) reconfigurable network.
\end{abstract}

\section{INTRODUCTION}

In the field of unconventional computing, the term "Chemical Computer" started spreading around the 90's [1]-[3]. A class of these computers is the spatially expanded biochemical computers [4], [5], which belongs to the greater family of reaction-diffusion computers [6]-[8] and have been the reference point for the inauguration of a continuous research in this direction [6]. In particular, a reaction-diffusion computer processes information by using excitation and diffusion waves which interact locally. A classic example that is also a source of inspiration for this paper, is the Belousov-Zhabotinsky (BZ) reaction, which is a non-equilibrium thermodynamic reaction exhibiting to nonlinear chemical oscillators. In these types of unconventional computing systems, information is encoded to the concentrations of the chemical species and the computation is performed by chemical reactions [5] or interactions of the excitation wave fronts [9].

The aforementioned computations emerge (Emerging Computing) through the spread and interaction of the entire wave fronts (Wave Computing [10]). A typical example of wave computing is the experimental realization of logic gates by the utilization of waves resulting from the proper chemical reactions within the chemical propagation medium [9], thus paving the way towards the development of unconventional information processing systems based on chemical wave propagation.

The Memristor is a two-terminal nanoscale electronic device originally introduced by Leon Chua in [11], which has been recently revisited in literature [12] and its potential applications are under investigation, in digital, analog, and mixed signal

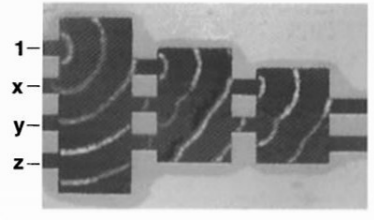

(a)

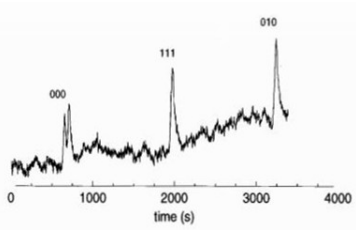

(b)
Fig. 1. (a) A 3-input logic chemical OR gate, demonstrated for the case of input $\left({ }^{1} 0^{1},{ }^{1} 0^{1},{ }^{1} 0^{1}\right)$. (b) Aggregate wave intensity measurement of the two outputs for the cases $\left({ }^{1} 0^{1}, 0^{1},,^{1} 0^{1}\right),\left({ }^{1} 1^{1}, 1^{1} 1^{1}, 1^{1}\right)$, and $\left({ }^{1} 0^{1}, 1^{1} 1^{1},{ }^{1} 0^{1}\right)$. Synchronized outputs correspond to ${ }^{1} 1^{1}$, while the two adjacent peaks are translated to output ${ }^{1} 0^{1}$. The ever increasing mean value is due to the aging of the system (adopted from [9]).

domains [13]-[17]. Memristor has been previously presented to introduce learning capabilities in common RLC circuits [18] and further used in oscillation-based circuits and systems able to solve computationally hard problems [19], [20] and emerge neuron-like behavior [21].

Previously, memristors have been successfully used by Asai and colleagues to implement a coupling between excitable elements [22], [23]. Propagation and interaction of excitation wave fronts have been demonstrated in their works using modified Oregonator model. In present paper we take a different route and represent an excitable element itself by a circuit with a memristor. In this work a Memristor-RLC (M-RLC) network configuration that mimics the behaviour of the chemical waves propagation has been designed to perform both conventional and unconventional calculations utilizing the same medium with a sense of reprogrammability. In this memristive network, two different computing functionalities are presented with examples. More specifically, the first one is the capability of the memristive network to implement wave based Boolean logic gates on the same medium under the appropriate voltage regulation providing the designers with a universal set of Boolean gates; while the second one, is mostly related on how this exact memristive network manages to perform neuron-like operations under the same voltage regulation thus presenting an appealing twofold unified computing approach. This echoes with living neural networks which often exhibit switches in the functionality. Thus, for example, a direction synaptic activity of spatially close neuronal ensembles in superior parietal lobule, which imitate a topology of a maze, have been reported in experiments with maze tracing by humans [24]. 


\section{Chemical Wave Gates}

Chemical Wave Gate paradigms were firstly presented in [9], where reaction-diffusion experiments were performed with a BZ medium where Boole logic operations were carried out through the propagation of oxidation wave-fronts. The presented chemical wave logic was based on the specific geometry of a BZ reaction space and at the constant speed propagation of chemical waves, initiated by external impulses (inputs). The geometry of a particular gate determines the nature of its operation and can be modified by appropriate external manipulation.

As an example, in Fig. 1, a chemical gate with the OR gate geometry is presented [9]. It has been made with inputs where the presence or absence of excitation at inputs $x, y$, and $z$ corresponds to 1 and 0 , accordingly. There is an additional input called 1 and its purpose is to generate a wave even in the case where the rest inputs are $(0,0,0)$, as well as for the synchronization of the inputs. The output is defined as true or false depending on whether the waves exit synchronously or asynchronously from the two channels on the right.

\section{ElECtRonic EQUiVALENT MODEL OF CHEMICAL MEDIUM UTILIZING M-RLC CIRCUITS}

To mimic the behaviour of the chemical gates, the first step was to design an electronic circuit capable of propagating waves. It was noticed that RLC circuits, acting as the equivalent circuit of transmission lines (Fig. 2(a)), fulfill the necessary criteria to implement an electronic wave propagation medium corresponding to the $\mathrm{BZ}$ membrane. The local nonlinearity, of the BZ medium, has been further integrated in the RLC modules of the transmission line by the introduction of memristor's nonlinear dynamics. The memristor dynamics' equations used in this work derived from the memristor model presented in [25], leading to a novel modified version of RLC modules, namely the M-RLC shown in Fig. 2(b). In terms of generality and directly associated with the desired computing reprogrammability, the voltage source $V_{S}$ is included in the MRLC module, however its use is prominent only for the case of neuron-like operations as examined later, otherwise is set to OV (ground).

In particular, each end of the M-RLC module is connected to a neighboring one forming a two-dimensional transmission line (Fig. 2(c)). An applied voltage at any edge, or intermediate module, of this grid is afterwards propagating through the medium, so the voltage across the capacitor $V_{C}$ is measured in order to visualize this propagation. In respect to the network's boundary conditions, in order to get a close affinity to the chemical gate paradigms, an annihilation of the excitation wave-front at the edges of the network is achieved by placing grounding resistors at the edges, with resistance values equal to the characteristic impedance of the grid. The speed of the wave in the M-RLC network is equal to $\mathrm{v}=10.000$ nodes $/ \mathrm{s}$.

\section{M-RLC WAVE Gates}

Aiming to verify the functionality of the proposed electronic approach in accordance with the chemical paradigms in [9],

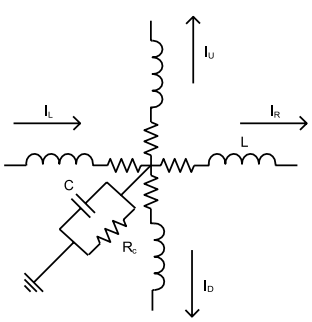

(a)

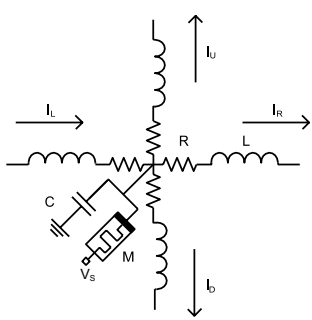

(b)

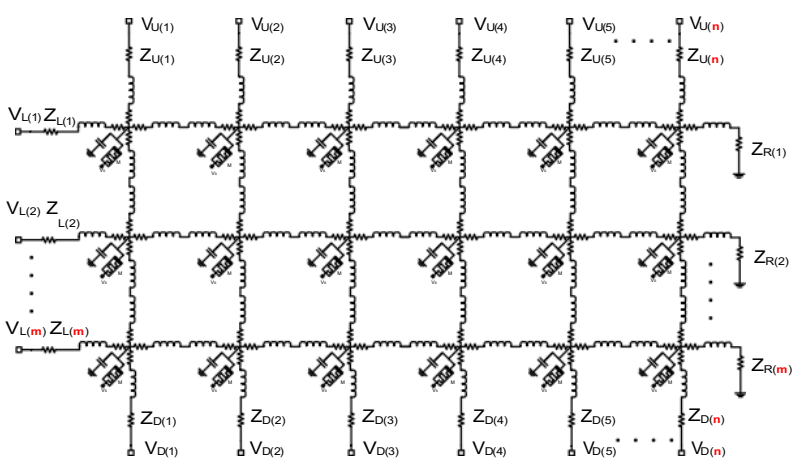

(c)

Fig. 2. (a) $2 \mathrm{D}$ transmission line RLC equivalent and (b) the proposed M-RLC modified configuration. In both cases the parameters of the passive elements are $\mathrm{R}=0.01 \Omega, \mathrm{L}=1 \mathrm{mH}, \mathrm{C}=10 \mathrm{~F}$. (c) Generic $2 \mathrm{D}$ grid of interconnected M-RLC modules.

the 2-input logic gates NOR and XNOR were designed. In the proposed approach, the logic gates were implemented by a $111 \times 100 \mathrm{M}-\mathrm{RLC}$ grid separated in two different areas, as shown in Fig. 3, one fully connected and one that forms the two thin output channels following the chemical experiments. The fully connected area allows the propagation of the input signals in the medium, while the second one, which only utilizes two straight paths of $5 \times 50 \mathrm{M}-\mathrm{RLC}$ modules each and the rest (black) remain unconnected, restricts the incoming front of the waves and drives it to the output. The time difference between the incoming waves in the output channels is remaining constant and defines the logical output of the gate, in correspondence to the chemical gates' output mechanism.

The architecture of the computing medium remains exactly the same for the case of NOR and XNOR gates; however, the unique required modification to perform different gate operation is the positioning of the input signal. In both cases, the always-active signal 1 is located in equidistant point from either output channel, giving the desired synchronized output in case of the absence of both input signals. On the other hand, the position of the two input signals differs, as for the NOR gate $\{x, y\}$ are located in two neighboring M-RLC modules at the one side of the signal 1 excitation location, while for the XNOR gate $\{x, y\}$ are located in two M-RLC modules equally distant from the signal 1 , but in the opposite side. The excitation of the M-RLC grid and the time difference of the propagating waves into the output channels as well the exact locations of the inputs are presented in Fig. 3, for all the possible combinations of input signals $\{x, y\}$, respectively.

\section{$M-R L C N A N D$ wave gate}




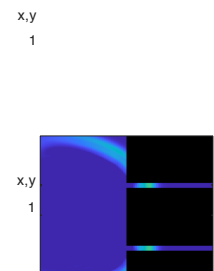

(a) $\{0,0\}$ $\mathrm{x}, \mathrm{y}$

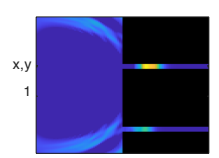

(b) $\{0,1\}$
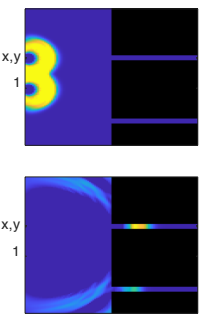

(c) $\{1,0\}$
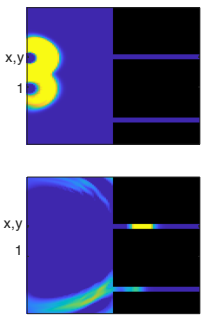

(d) $\{1,1\}$
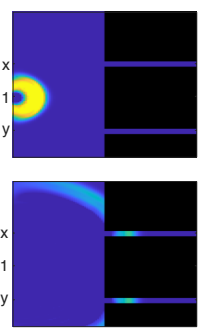

(e) $\{0,0\}$
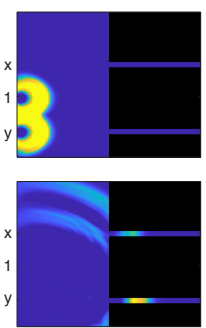

(f) $\{0,1\}$
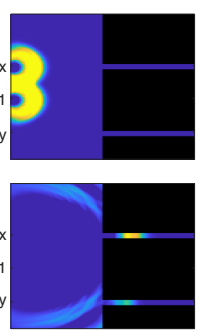

(g) $\{1,0\}$
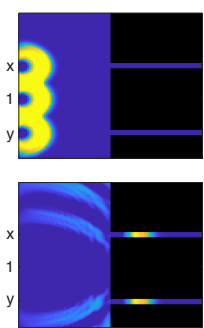

(h) $\{1,1\}$

Fig. 3. Excitation (top) and output (bottom) frames of wave propagation in the NOR (a)-(d) and XNOR (e)-(h) gates for every input $\{x, y\}$ combination.

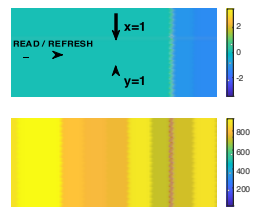

(a) $t_{1}$

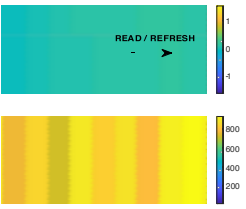

(b) $t_{2}$

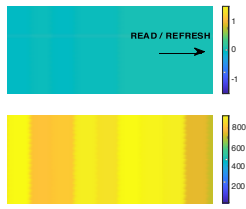

(c) $t_{3}$
Fig. 4. $V_{C}[V]$ of each M-RLC module (top) and Memristance values $M[\Omega]$ (bottom) for 3 different timesteps of the proposed NAND gate's operation for $\{x, y\}=\{1,1\}$.

Given that the aforementioned examples do not require the utilization of memristor's nonlinear dynamics, the amplitude of the input signals was always low enough to not modify the initial high resistance state of the memristor. However, the output mechanism of the previous paradigms, which is utilizing the timing difference of the two waves, is not very practical for the further cascadability of the gates to more complex Boolean functions. Thus, a novel methodology for the implementation of wave propagation based logic gates, with the same medium as above that exploits memristor's dynamics, is presented. In this approach, the output of the logic gate is defined as the presence (logical 1) or the absence (logical 0 ) of strong negative amplitude on output loads $\left(Z_{R, 1: m}\right)$ at specific time.

In details, a 2-input M-RLC NAND gate configuration is proposed, where the gate operation results from two different events, the read and the write. Both of them are initiated simultaneously but they are taking place in different area of the grid. The write event constitutes the effect of the input signals $y$ the resistance values of the memristors in a specified area of the grid, far from the location of the read signal. While, the read event performs the read and refresh processes of the gate's state.

In such a way, the input signals $\{x, y\}$ are applied to a short area of the $V_{U}$ and $V_{D}$, respectively, such as half- period negative sinusoidal signals, which are able to change the memristance values of some M-RLC modules when they are interfering. In specific, when none or one of them is applied, it is not strong enough to modify the initial high resistance state of the memristor, but when two of them interfere, the meristance can change towards the low resistance state. Furthermore, when memristors are in low resistance state, the central node of the M-RLC is practically grounded, so the damping throughout the M-RLC grid is strong.

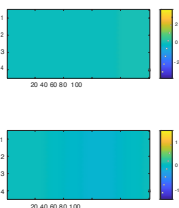

(a) $\{0,0\}$

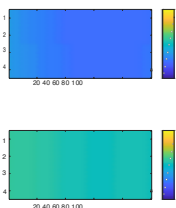

(b) $\{0,1\}$

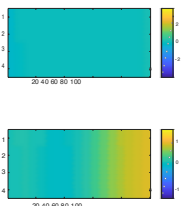

(c) $\{1,0\}$

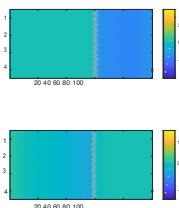

(d) $\{1,1\}$
Fig. 5. $V_{C}[V]$ of each M-RLC at (top) excitation ( $\left.t_{1}\right)$ and (bottom) the output $\left(t_{3}\right)$ frame, for every input $\{x, y\}$ combination.

At the same time, a full-period sinusoidal signal, with phase delay of 180 degrees, is applied at the $V_{L, 1: m}$, corresponds to the read event, and travels through the medium. The amplitude of the negative half of the reading pulse is not enough to change memristor's state to low impedance. In case that some memristors of the medium are already in low resistance state, due to the interference of the inputs, the incoming negative half of the reading signal is not able to reach the output loads (logical 0 ). Then, the positive half of the reading signal, which is able to change back every memristor's state to high resistance, travels through the medium and prepares it for the next logic operation.

As an example, Fig. 4 illustrates the operation of the wave gate for the case of both inputs are applieq $x,\} y=\{1,1\}$. Firstly, in Fig. 4(a) the input signals are applied (top) and modified the memristance state of an area in the grid (bottom), while the read/refresh signal starts its propagation through the medium. Then, in Fig. 4(b) the highly damped negative half of the read/refresh signal is evident, while the positive half is switching back the memristor's state to high impedance. Finally, the Fig. 4(c) presents the weak negative signal that reaches the output loads and the grid's memristance states that are completely clear for the next logic operation. Moreover, the corresponding $t_{1}$ (top) and $t_{3}$ (bottom) frames for all the input combinations are presented in Fig. 5, showing the negative half of the reading signal reaching the output loads in all cases apart from the $\{x, y \neq 1,\{1$. The NAND gate is a universal gate characterized by the property of functional completeness.

In addition, the memristors of the M-RLC grid can operate as information storage and attribute further capabilities to the proposed gate.

\section{Neuron-Like Behaviour of THE M-RLC}

Utilizing the adaptability of the proposed medium, the duality of its nature to perform either as conventional computing 


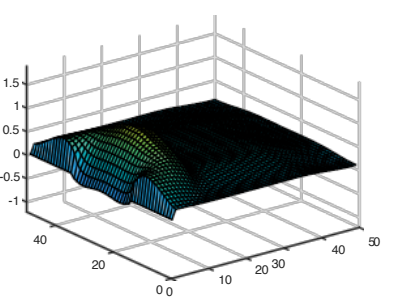

(a) $V_{C, t_{1}}$

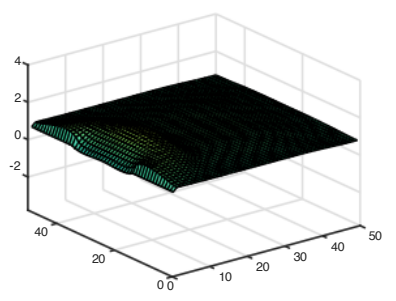

(e) $V_{M, t_{1}}$

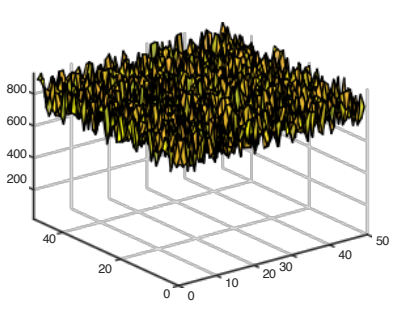

(i) $M_{t_{1}}$

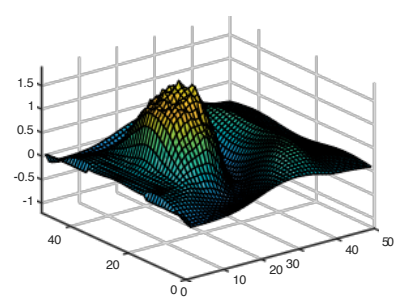

(b) $V_{C, t_{2}}$

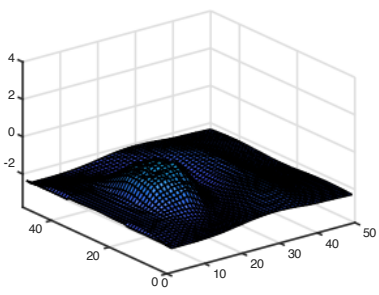

(f) $V_{M, t_{2}}$

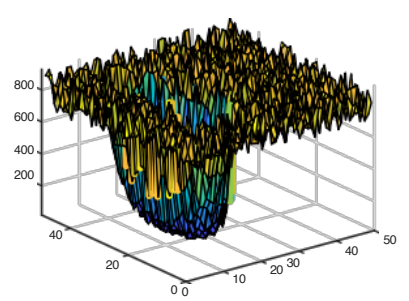

(j) $M_{t_{2}}$

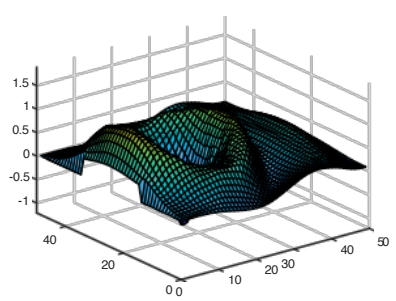

(c) $V_{C, t_{3}}$

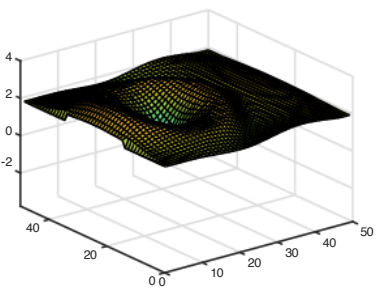

(g) $V_{M, t_{3}}$

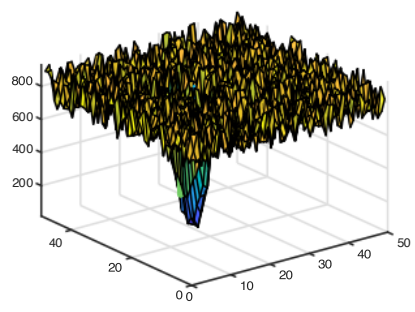

(k) $M_{t_{3}}$

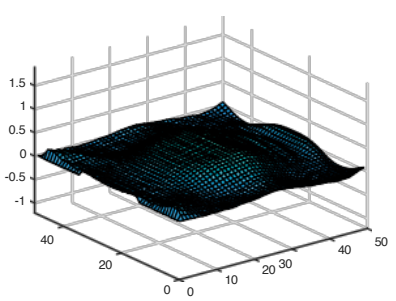

(d) $V_{C, t_{4}}$

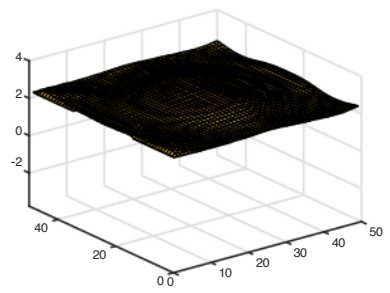

(h) $V_{M, t_{4}}$

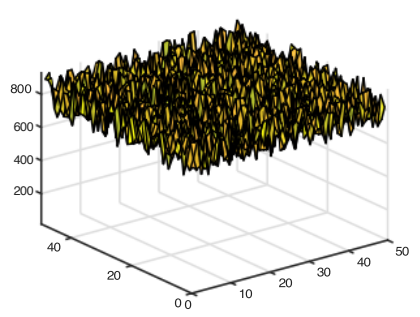

(1) $M_{t_{4}}$

Fig. 6. Time evolution of $V_{C}[V]$ (a)-(d), $V_{M}[V]$ (e)-(h), and $M[\Omega]$ (i)-(l) during a complete neuron-like operation in the proposed medium.

element (logic gate) or as a more complex unconventional computing element (i.e. neuron) is here investigated. Thus, in this approach we are examining the ability of the proposed M-RLC grid to produce neuron-like spikes. These spikes are emerging from the combination of the external inputs (signals applied to the edge of the grid), the state of the memristor devices and the applied voltage through the $V_{S}$ (sinusoidal signal of $3 \mathrm{~V}$ amplitude), common for every M-RLC module of the grid. First of all, each memristor state is essential for the effect of the $V_{S}$ signal to the M-RLC grid, as a high resistance memristor induces high voltage drop of $V_{S}$ 's amplitude. In such a way, a M-RLC grid, initialized at high memristance states (Fig. 6, $t_{1}$ ), prevents $V_{S}$ signal from reaching the corresponding $V_{C}$ nodes and as a result the M-RLC grid. However, $V_{S}$ applies a high amplitude sinusoidal voltage at the top electrode of the memristors and in combination with an external signal that propagates in the medium can affect the memristors' state. In particular, while $V_{S}$ amplitude is not enough to change the memristors' state, if $V_{S}$ is at the positive half and an external signal at the negative half of the sinusoidal, the voltage across the memristor is accumulated and its state is changing towards the low memristance state (Fig. 6, $t_{2}$ ). Finally $V_{S}$ reaches the medium and generates a strong pulse that afterwards is spreading (Fig. 6, $t_{3}$ ). In the meantime, $V_{S}$ is able to switch memristors' state back to high resistance and the medium lies at the resting phase (Fig. 6, $t_{4}$ ), until a next external input is able to activate the medium again.

\section{Conclusions}

This work aims to merge the conventional and the unconventional calculations utilizing a single medium, consisting of many identical interconnected M-RLCs. Through electric wave propagation Boolean calculations and neuron-like behaviour have been demonstrated. Additionally, a particularly useful feature of the proposed electronic circuit structure is that the electronic wave propagation medium exhibits reprogramming abilities by utilizing memristors adaptivity. This extends to the realization of different logic operations on the same medium under the appropriate voltage regulation, resulting in the implementation of a universal set of logical gates capable of implementing any logic function (universality) and the representation of neuron-like spiking behaviour of the M-RLC memristive network. As a future work, power, space analysis and the cascadability of the M-RLC grid will be examined together with the effect of memristors' variability, as well as more accurate, corresponding to fabricated devices, memristor models will be tested towards to the physical implementation of the proposed memristive network. 


\section{REFERENCES}

[1] A. Hjelmfelt, E. D. Weinberger, and J. Ross, "Chemical implementation of neural networks and turing machines," Proceedings of the National Academy of Sciences, vol. 88, no. 24, pp. 10983-10 987, 1991.

[2] A. Hjelmfelt, E. D. Weinberger, and J. Ross, "Chemical implementation of finite-state machines," Proceedings of the National Academy of Sciences, vol. 89, no. 1, pp. 383-387, 1992.

[3] A. Hjelmfelt and J. Ross, "Chemical implementation and thermodynamics of collective neural networks," Proceedings of the National Academy of Sciences, vol. 89, no. 1, pp. 388-391, 1992.

[4] L. Kuhnert, "A new optical photochemical memory device in a lightsensitive chemical active medium," Nature, vol. 319, no. 6052, p. 393, 1986.

[5] L. Kuhnert, K. Agladze, and V. Krinsky, "Image processing using lightsensitive chemical waves," Nature, vol. 337, no. 6204, p. 244, 1989.

[6] A. Adamatzky, B. D. L. Costello, and T. Asai, Reaction-diffusion computers. Elsevier, 2005.

[7] A. Adamatzky, "Physarum machines: encapsulating reaction-diffusion to compute spanning tree," Naturwissenschaften, vol. 94, no. 12, p. 975, 2007.

[8] A. Adamatzky, G. J. Mart'inez, L. Zhang, and A. Wuensche, "Operating binary strings using gliders and eaters in reaction-diffusion cellular automaton," Mathematical and Computer Modelling, vol. 52, no. 1-2, pp. 177-190, 2010.

[9] O. Steinbock, P. Kettunen, and K. Showalter, "Chemical wave logic gates," The Journal of Physical Chemistry, vol. 100, no. 49, pp. 18970 $18975,1996$.

[10] T. Roska, "Computational and computer complexity of analogic cellular wave computers," Journal of Circuits, Systems, and Computers, vol. 12 no. 04, pp. 539-562, 2003.

[11] L. Chua, "Memristor-the missing circuit element," IEEE Transactions on Circuit Theory, vol. 18, no. 5, pp. 507-519, September 1971.

[12] D. B. Strukov, G. S. Snider, D. R. Stewart, and R. S. Williams, "The missing memristor found," nature, vol. 453, no. 7191, p. 80, 2008.

[13] L. Chua, G. Sirakoulis, and A. Adamatzky, Handbook of Memristor Networks. Springer International Publishing, 2019.

[14] G. Papandroulidakis, I. Vourkas, N. Vasileiadis, and G. C. Sirakoulis, "Boolean logic operations and computing circuits based on memristors," IEEE Transactions on Circuits and Systems II: Express Briefs, vol. 61, no. 12, pp. 972-976, 2014.

[15] Y. V. Pershin and M. Di Ventra, "Practical approach to programmable analog circuits with memristors," IEEE Transactions on Circuits and Systems I: Regular Papers, vol. 57, no. 8, pp. 1857-1864, 2010.

[16] B. Li, Y. Wang, Y. Wang, Y. Chen, and H. Yang, "Training itself: Mixedsignal training acceleration for memristor-based neural network," in Design Automation Conference (ASP-DAC), 2014 19th Asia and South Pacific. IEEE, 2014, pp. 361-366.

[17] R. Tetzlaff, Memristors and memristive systems. Springer, 2013.

[18] V. Ntinas, I. Vourkas, and G. C. Sirakoulis, "LC filters with enhanced memristive damping," in Circuits and Systems (ISCAS), 2015 IEEE International Symposium on. IEEE, 2015, pp. 2664-2667.

[19] V. Ntinas, I. Vourkas, G. C. Sirakoulis, and A. I. Adamatzky, "Oscillation-based slime mould electronic circuit model for mazesolving computations," IEEE Transactions on Circuits and Systems I: Regular Papers, vol. 64, no. 6, pp. 1552-1563, 2017.

[20] V. Ntinas, I. Vourkas, G. C. Sirakoulis, and A. I. Adamatzky, "Modeling physarum space exploration using memristors," Journal of Physics D Applied Physics, vol. 50, no. 17, 2017.

[21] V. Ntinas, I. Vourkas, G. C. Sirakoulis, A. Adamatzky, and A. Rubio, "Coupled physarum-inspired memristor oscillators for neuron-like operations," in Circuits and Systems (ISCAS), 2018 IEEE International Symposium on. IEEE, 2018, pp. 1-5.

[22] X. Gong, T. Asai, and M. Motomura, "Reaction-diffusion media with excitable oregonators coupled by memristors," in Cellular Nanoscale Networks and Their Applications (CNNA), 2012 13th International Workshop on. IEEE, 2012, pp. 1-6.

[23] T. Asai, "Reaction-diffusion media with excitable oregonators coupled by memristors," in Memristor Networks. Springer, 2014, pp. 625-635.

[24] P. Gourtzelidis, C. Tzagarakis, S. M. Lewis, D. A. Crowe, E. Auerbach, T. A. Jerde, K. Uğurbil, and A. P. Georgopoulos, "Mental maze solving: directional fMRI tuning and population coding in the superior parietal lobule," Experimental brain research, vol. 165, no. 3, pp. 273-282, 2005.
[25] I. Vourkas and G. C. Sirakoulis, "A novel design and modeling paradigm for memristor-based crossbar circuits," IEEE Transactions on Nanotechnology, vol. 11, no. 6, pp. 1151-1159, 2012. 